\title{
The Effect of Clay/Multiwall Carbon Nanotube Hybrid Fillers on the Properties of Elastomer Nanocomposites
}

\author{
Sung Ho Song (iD) \\ Division of Advanced Materials Engineering, Kongju National University, Chungnam 330-717, Republic of Korea \\ Correspondence should be addressed to Sung Ho Song; shsong805@gmail.com
}

Received 16 November 2017; Revised 19 February 2018; Accepted 8 March 2018; Published 15 April 2018

Academic Editor: Shunsheng Cao

Copyright (C) 2018 Sung Ho Song. This is an open access article distributed under the Creative Commons Attribution License, which permits unrestricted use, distribution, and reproduction in any medium, provided the original work is properly cited.

\begin{abstract}
The hybrid fillers of 1D multiwalled carbon nanotubes (MWNT) and 2D montmorillonite (MMT) have led to excellent physical and chemical properties in high performance elastomer nanocomposites. In this study, the hybridization of PDDA (polydiallyldimethylammonium chloride) functionalized MWNT (P-MWNT) and hydroxyl-functionalized MMT (H-MMT) was prepared by the electrostatic interaction between the positive charge on the MWNT and the negative charge on the MMT using a simple solution mixing process. Also, a styrene-butadiene rubber (SBR) nanocomposite containing the hybrid nanofillers was prepared to improve the dispersion of nanofillers with SBR latex. The SBR nanocomposites with the hybrid nanofillers exhibited outstanding mechanical properties including modulus, tensile strength, and elongation at break, due to the enhanced interfacial bonding with the elastomer matrix. Furthermore, the hybrid nanofillers in the SBR matrix showed superior thermal and electrical properties and gas barrier performance at low loadings. The synergistic effects of the SBR produced by the hybridized nanofillers will open up new opportunities for elastomer composites with high performance.
\end{abstract}

\section{Introduction}

Elastomers which have the ability to withstand large strain and exhibit high viscoelastic properties are widely applied in tires, impact bumpers, belts, and packaging. However, to meet the needs of industrial applications, elastomers were reinforced by adding various nanofillers because most neat elastomers were afflicted with low mechanical strength [1-3]. Generally, carbon black (CB) is added as a reinforcing filler in the elastomer industry [4]. However, the CB tends to form agglomerates in the elastomer matrix due to strong van der Waal forces, and this reduces their reinforcing performance in the elastomer composites [5]. To solve this problem, a lot of researches and efforts have concentrated on the development of high performance elastomer nanocomposites by the incorporation of nanofillers such as carbon nanotubes $[6,7]$, layered silicate [8-11], graphene [12], and calcium carbonate [13-15]. Also, because the properties of elastomer composites can be finely tuned by the nanofillers, the combination of nanofillers and elastomer matrices has also attracted significant scientific interest.
Among these nanofillers, carbon nanotubes (CNTs) and montmorillonite (MMT) have shown attractive fillers in tire tread compounds because they improve the tread grip and rolling resistance performance of the compound at low temperature [16, 17]. CNTs have high flexibility, low mass density, high tensile strength $(150 \sim 200 \mathrm{GPa})$, large aspect ratio, and high electrical $\left(\sim 10^{6} \mathrm{~S} / \mathrm{cm}\right)$ and thermal conductivity $(\sim 6,000 \mathrm{~W} / \mathrm{mK})[6,7]$. Also, montmorillonite (MMT), which are inexpensive and substantially available, make interesting reinforcing fillers due to large surface area and high aspect ratio of layered silicates. Furthermore, incorporating the hybrid nanofillers with two geometrically dissimilar nanomaterials as reinforcing fillers is more interesting in the elastomer matrix owing to its significant synergistic effects $[18,19]$.

For example, Zhang et al. exhibited that the tensile strength of PVA (poly(vinyl alcohol)) composites containing CNTs-graphene hybrids (0.6 wt $\%$ ) was $34 \%$ and $20 \%$ higher than those of nanocomposites with $0.6 \mathrm{wt} \%$ graphene and CNTs alone, respectively [20]. These reasons are that the fillers are uniformly codispersed by the formation of 
TABLE 1: Formulation of the elastomer nanocomposites.

\begin{tabular}{lcccc}
\hline Materials & Reference & MMT & MWNT & MMT-MWNT \\
\hline SBR & 80 & 80 & 50 & 80 \\
Carbon black & 50 & 50 & 1 & 50 \\
Stearic acid & 1 & 1 & - & - \\
MMT & - & - & 5 & - \\
MWNT & - & - & - & 5 \\
Solution mixed & - & 1.75 & 1.75 & 1.75 \\
MMT-MWNT & 1.75 & 1 & 1 & 1 \\
Sulfur & 1 & - & \\
TBBS & 1 & & \\
\hline
\end{tabular}

MWNT: multiwall carbon nanotube; MMT: montmorillonite clay; TBBS: N-tert-butyl-2- benzothiazyl sulfonamide; MMT-MWNT: hydroxyl-functionalized exfoliated montmorillonite/polydiallyldimethylammonium chloride- (PDDA-) modified-MWNT.

interconnected filler networks in the elastomer matrix. Zhao et al. fabricated CNTs/MMT hybrid nanofillers based PVA nanocomposites by solution mixing method. The storage modulus was enhanced by $133 \%$, following the addition of CNTs/MMT (7 wt\%), compared with neat PVA [21]. Also, Tang and coworkers announced a synergistic effect for CNTs/clay hybrid nanofillers due to the formation of a filler network in chitosan matrix [22].

Nevertheless, while the performance improvement of elastomer nanocomposites by the synergetic effects of two different nanofillers has been of great interest, few works have been reported on the synthesis and properties of the CNTs/MMT/elastomer hybrid system by latex mixing.

In the present study, we demonstrated the remarkable synergistic effect of CNTs/MMT hybrid nanofillers in elastomer matrix prepared by just simple solution mixing. These hybrid nanofillers were prepared with an electrostatically interacting assembly of polydiallyldimethylammonium chloride- (PDDA-) modified-MWNT (P-MWNT) and hydroxyl-functionalized exfoliated montmorillonite $(\mathrm{H}-$ MMT) by using a simple solution method. Furthermore, in this work, a master-batch of SBR was prepared to enhance the dispersion of the hybrid nanofillers in the styrene-butadienerubber (SBR) latex matrix. The latex mixing method is environmentally friendly and scalable and shows great opportunities for enhancing the performance of elastomer nanocomposites in various applications. The formation of a hybrid filler network was facilitated by the strong interaction between the P-MWNT and H-MMT in the elastomer matrix.

Furthermore, the interfacial interaction between the hybrid nanofillers and SBR was further enhanced by incorporating CNTs into the interfacial region. The resulting $\mathrm{P}$ MWNT/H-MMT hybrid nanofillers exhibited remarkable synergetic effects which significantly enhanced the mechanical, thermal, and electrical properties of the elastomer nanocomposites.

\section{Experimental Section}

2.1. Materials. The matrix material was selected as styrene butadiene rubber (SBR 1500, Kumho Petrochemical Co. Ltd., Korea) consisting of styrene of $23 \%$ and butadiene of
77\%. The carbon black (N-330, OCI Co. Ltd., Korea) and N-tert-butyl-benzothiazole sulfonamide (TBBS, Shangdong Shanxian chemical Co. Ltd., China) were used and zinc oxide $(\mathrm{ZnO})$, stearic acid (S/A), and sulfur were purchased from Sigma-Aldrich. A multiwall carbon nanotube (MWNT) was supplied by Hanwha NanoTech (Korea) and montmorillonite clay (MMT and Cloisite 30A) was purchased from Southern Clay Products (Australia).

2.2. Preparation of Hybrid Nanomaterials. The polydiallyldimethylammonium chloride- (PDDA-) modified-MWNT (P-MWNT) was synthesized by mixing PDDA (20 mg) and MWNT (20 mg) in N-methyl-2-pyrrolidone (NMP, $200 \mathrm{ml}$ ) under sonication. The ultrasonic treatment has been carried out for $1 \mathrm{~h}$. Then the mixture was performed at $80^{\circ} \mathrm{C}$ for $24 \mathrm{~h}$. For the preparation of the P-MWNT and H-MMT hybrid colloid, H-MMT exfoliated in water was added to the asprepared P-MWNT aqueous dispersion with the H-MMT and P-MWNT weight ratio of $1 / 1$, followed by sonication for 30 min, resulting in a homogeneous mixture dispersion of $\mathrm{H}$ MMT and P-MWNT.

2.3. Preparation of Elastomer Nanocomposites. The hybrid nanomaterials/SBR nanocomposites were prepared by following standard procedures in Table 1. First, the SBR latex (20 phr) with 5 phr (parts per hundred, rubber by weight) carbon black, H-MMT, P-MWNT, and hybrid nanomaterials $\mathrm{H}-\mathrm{MMT} / \mathrm{P}-\mathrm{MWNT}$ were mixed by vigorous stirring for $24 \mathrm{~h}$, respectively. During coagulation, butadiene-styrene-vinylpyridine rubber (VPR) was added to a small loading, and VPR not only plays a key role in the prevention of aggregation of H-MMT/P-MWNT but also acts as an interfacebridge between H-MMT/P-MWNT and SBR. The nanomaterials/SBR emulsion was then coagulated by a $1.0 \mathrm{phr}$ sulfuric acid solution. The coagulated composites were washed with water until the $\mathrm{pH}$ of the filtered water reached $6 \sim 7$ and then dried in an oven at $50^{\circ} \mathrm{C}$ for $24 \mathrm{~h}$. And then, the SBR 80 phr and carbon materials (5 phr)/SBR emulsion (20 phr) were mixed with 50 phr carbon black in Banbury mixer at a rotor speed of $60 \mathrm{rpm}$ for mater batches. The additives and vulcanization agents were added at the end so that curing process of the mixture could be started. The compounds 


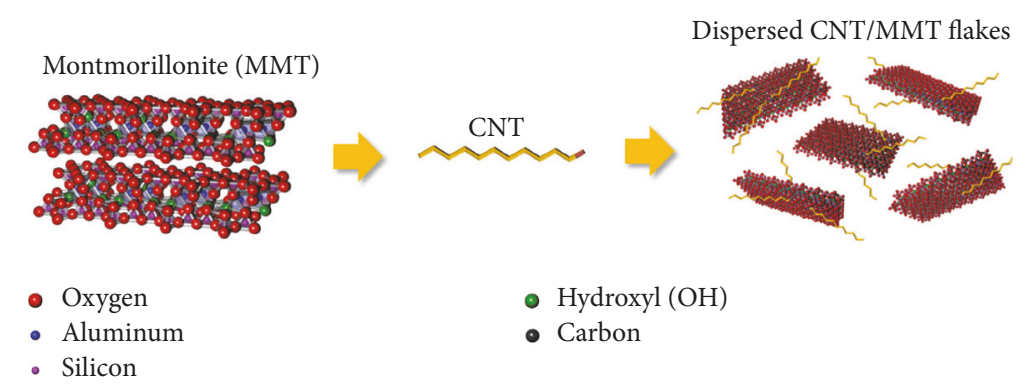

(a)

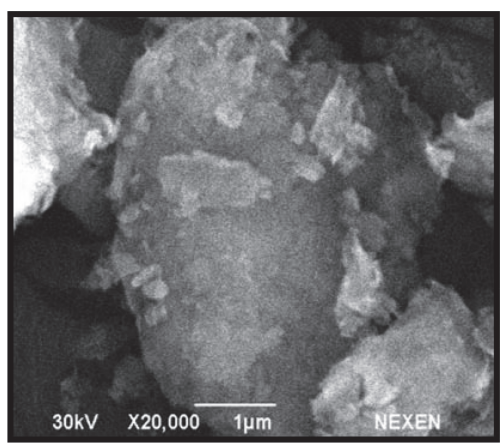

(b)

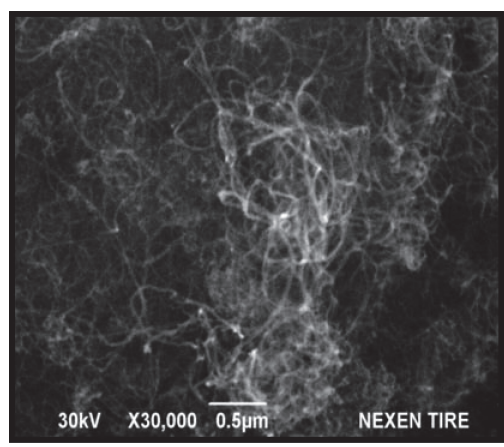

(c)



(d)

FIGURE 1: (a) Schematic diagram showing the overall processing of fabricated MMT/MWNT; ((b), (c), and (d)) SEM images of the MMT, MWNT, and MMT/MWNT, respectively.

were placed in the aluminum mold and cured at $160^{\circ} \mathrm{C}$ for $\mathrm{T}_{90}$ by rheometer under pressure. The formulations of the diver nanomaterials/SBR composites are summarized in Table 1 .

2.4. Characterization. The morphology of MMT, MWNT, and H-MMT/P-MWNT was investigated using fieldemission scanning electron microscopy (FE-SEM) (Hitachi S4800) analyses. Also, scanning electron microscopy (SEM, JEOL JSM-6490LV) was used to observe the morphology of the fractured surface of elastomer nanocomposites. The specimens were fractured in liquid nitrogen and the cross surface of samples was coated by gold using a sputtering process. Tensile tests were carried out in an Instron tensile machine (Instron Co., UK) at crosshead speed of $300 \mathrm{~mm} / \mathrm{min}$. The dumbbell shape samples were $10 \mathrm{~mm}$ in thickness and $5 \mathrm{~mm}$ in width (ASTM D- 412). At least four tests were carried out for each case. The $\mathrm{O}_{2}$ permeability was measured with ASTM Standard E-96 and a Mocon model DL 100 instrument. The values of the $\mathrm{O}_{2}$ transmission rate were obtained at $23^{\circ} \mathrm{C}$. The fatigue properties of composites were characterized by using Demattia (UESHIMA, ASTM D-3479). The thermal conductivity was characterized by placing the probe on the sample surface using the thermal conductivity analyzer (QTM-500, ASTM E1461). The electrical resistance of these manufactured composites was examined by the two-probe method in the TERAOHM (MI2077, ASTM D257) at room temperature.

\section{Results and Discussion}

The overall process of the proposed fabrication of $\mathrm{H}$ MMT/C-MWNT/elastomer composites is illustrated in Figure 1(a). The H-MMT/C-MWNT hybrid nanomaterials were synthesized by mixing polydiallyldimethylammonium chloride- (PDDA-) modified-MWNT (P-MWNT) with hydroxyl-functionalized exfoliated montmorillonite (HMMT). Then the elastomer nanocomposite using SBR latex with H-MMT/C-MWNT hybrids was synthesized by solution mixing method. Finally, various samples of SBR (80 phr) with 5 phr (parts per hundred, rubber by weight) CB (reference), H-MMT (MMT), P-MWNT (MWNT), and solution mixed H-MMT/P-MWNT (MMT-MWNT) in SBR latex (20 phr) were prepared, respectively. Additives and vulcanization agents were added at the end so that curing process of the mixture could be started (see details in Experimental Section). In Figure 1(b), SEM images exhibited 2D ultrathin MMT sheets with a lateral dimension of more than $1 \mu \mathrm{m}$, with morphologically irregular shapes. Figure 1(c) shows highly entangled and randomly organized CNT. Furthermore, it can be seen that the surface of the MMT layers adsorbs a large amount of MWNT in Figure 1(d). The connection between the MWNT and the MMT sheets can be obviously observed, and the MWNTs were randomly distributed on the surface of MMT.

SBR nanocomposites with MMT/MWNT produced by latex mixing were prepared with different nanomaterials for 


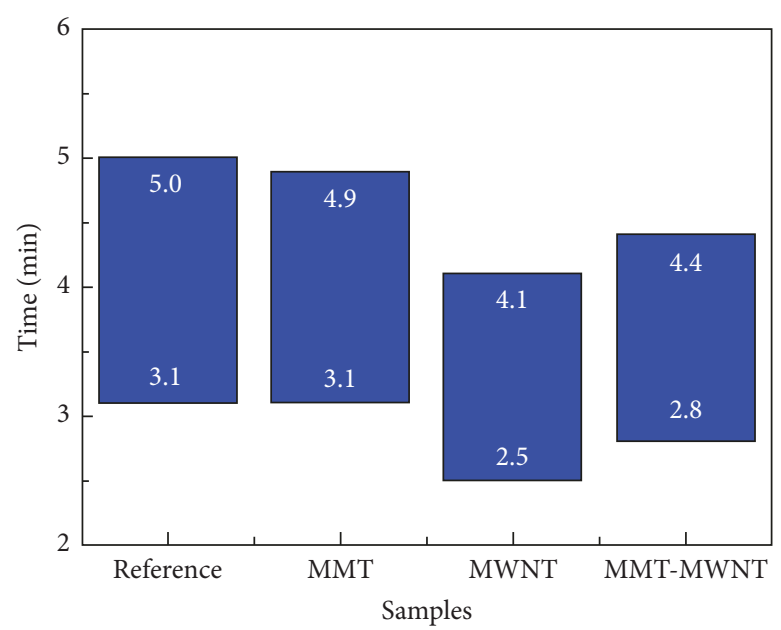

(a)

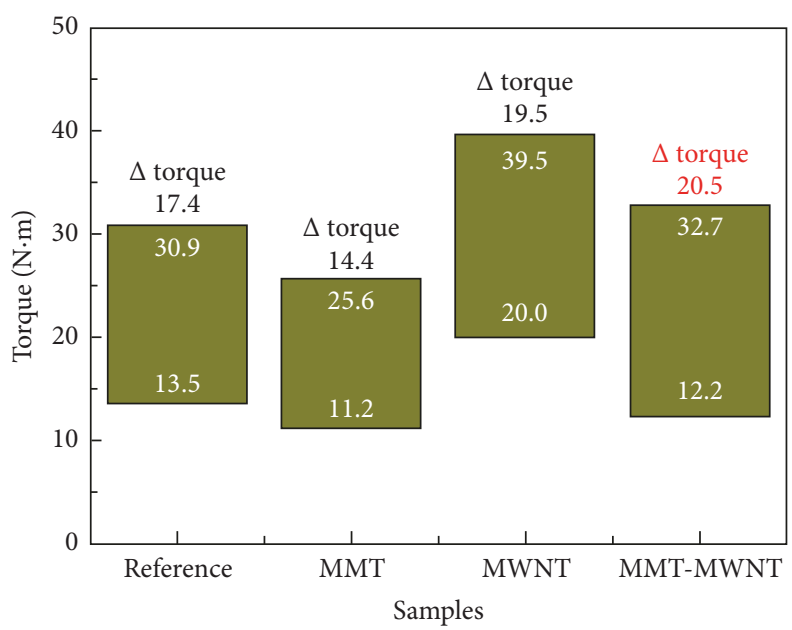

(b)

FiguRE 2: (a) Curing properties of elastomer nanocomposites with diverse fillers. (b) Torque of elastomer nanocomposites. (c) Stress-strain curves of the elastomer nanocomposites with diverse fillers. (d) Elastic modulus and toughness of the elastomer nanocomposites with diverse fillers.

TABLE 2: Mechanical properties of the elastomer nanocomposites.

\begin{tabular}{lcccc}
\hline & Reference & MMT & MWNT & MMT-MWNT \\
\hline Elongation (\%) & $400 \pm 12$ & $410 \pm 10$ & $315 \pm 11$ & $375 \pm 15$ \\
\hline
\end{tabular}

comparison with SBR (reference). The cure characteristics based on the scorch time $\left(t_{40}\right)$ and cure time $\left(t_{90}\right)$ of different nanomaterial-filled elastomer vulcanizates are shown in Figure 2(a). The values of the scorch and cure times of the MMT/MWNT/SBR composites are shorter than those of other elastomer composites due to strong interactions with the elastomer, which are especially strong with a nonpolar elastomer such as SBR [23]. A comparison of torque for SBR composites with different embedded fillers was showed in Figure 2(b). The $M_{H}-M_{L}$ ( $\Delta$ torque) value $(20.5 \mathrm{~N} \cdot \mathrm{m})$ of the SBR nanocomposites with MMT/MWNT, the crosslink density of vulcanization, was meaningfully increased compared to those of the other fillers nanocomposites $[2,22]$. This result indicates that the MMT/MWNT fillers resulted in stronger crosslinking with the SBR during vulcanization and supports the improved mechanical properties of the SBR nanocomposites shown in Figure 3. The physical properties of the MMT/MWNT/SBR nanocomposites were examined, along with nanocomposites based on other embedded materials. Figure 3(a) shows the modulus and tensile strength of the SBR nanocomposites with different integrated nanomaterials. The modulus of the nanocomposites with MMT/MWNT showed an increase by as much as $23 \%$ and $43 \%$ relative to that of reference and MMT nanocomposites, respectively. This performance enhancement was due to the strong interfacial bonding and improved dispersion of the sheets within the elastomer matrix. SEM images of the fracture surfaces of the elastomer nanocomposites after tensile tests are shown in Figures 3(c)-3(f). The SBR nanocomposite with MMT/MWNT exhibits roughened fractured surface, indicating the stronger interfacial adhesion between the MMT/MWNT and SBR matrix. The elongation at the break of the MMT/MWNT/SBR (375\%) shows it has enhanced properties compared with those of MWNT/SBR (315\%), shown in Table 2. The increased reinforcement can be attributed to the large contact area between the MMT/MWNT and the elastomer matrix in the SBR composite, as well as the coagulation of the MMT/MWNT and SBR via surface functional groups, which enhanced the interfacial adhesion and restricted the motion of the elastomer segmental chains. Figure 3(b) also shows a comparison of elastic modulus and toughness of the MMT/MWNT/SBR nanocomposites compared with those of other materials composites. The elastic modulus of the MMT/MWNT SBR nanocomposites was $0.33 \mathrm{MPa}$, which is higher than other results. The toughness (area under the stress-strain curve) of the reference and the MMT/MWNT nanocomposite ( $5 \mathrm{phr}$ ) was $5.71 \mathrm{MJ} / \mathrm{m}^{3}$ and $9.28 \mathrm{MJ} / \mathrm{m}^{3}$, respectively; the S-MM/SBR nanocomposite exhibited a toughening effect as high as $63 \%$. The elastic modulus and toughness of the MMT/MWNT/SBR nanocomposite at $5 \mathrm{phr}$ loading are much higher than those of other composites. This indicates that an applied mechanical load may transfer to MMT/MWNT through interfacial interactions [24]. The SEM images of the fracture elastomer composites are exhibited in Figures 3(c)-3(f). The MMT/MWNT/SBR nanocomposites exhibit roughened fractured surface, indicating the stronger interfacial adhesion between MMT/MWNT and SBR matrix. Also, the MMT/MWNT nanomaterials dispersed in elastomer matrix are showed in SEM images. 


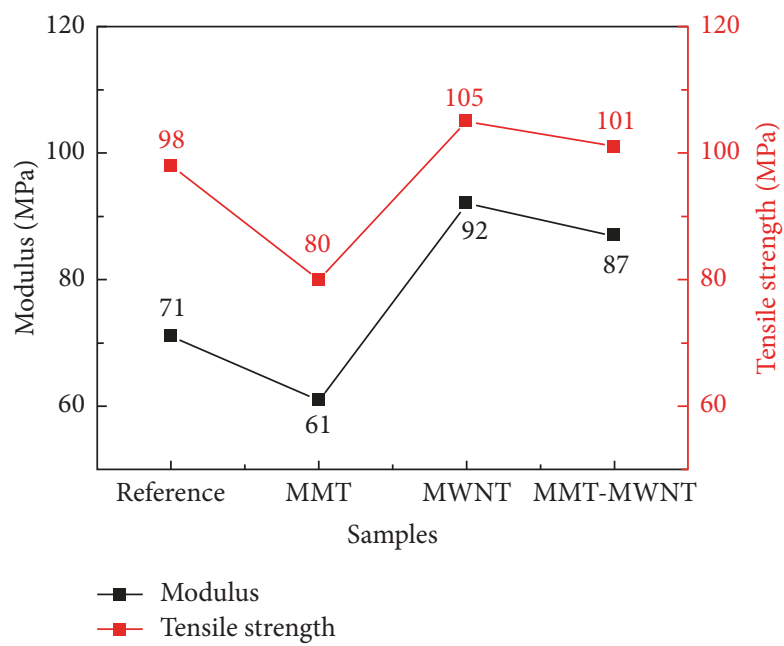

(a)



(c)



(d)

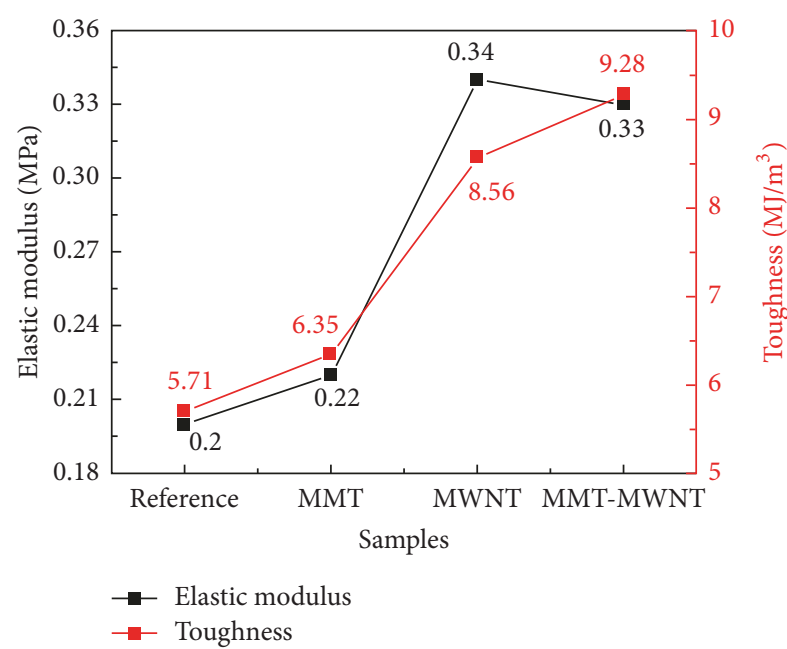

(b)



(e)

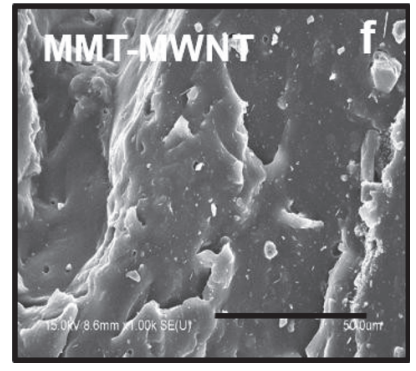

(f)

FIGURE 3: Mechanical properties of elastomer nanocomposites. (a) Stress-strain curves of the elastomer nanocomposites with diverse fillers. (b) Elastic modulus and toughness of the elastomer nanocomposites with diverse fillers. ((c), (d), (e), and (f)) SEM images of fracture surface of elastomer nanocomposites with reference, MMT, MWNT, and MMT/MWNT, respectively.

In Figure 4(a), the fatigue properties of the SBR nanocomposites were measured after 2,000, 6,000, and 10,000 cycles. Fatigue crack growth $(d c / d n)$ equation [23]:

$$
\frac{d c}{d n}=A \cdot G \alpha,
$$

where $c$ is the crack length, $n$ is the cycles, and $G$ is the tear energy. This equation means that fatigue crack growth is proportional to the tear energy, and (1) indicates that when tearing energy is decreased, fatigue life is increased. The MMT/MWNT sample exhibited a remarkable reduction in crack length, even after 10,000 cycles, and the fatigue crack growth of the MMT/MWNT (3.53) was decreased by over $142 \%$ compared to that of the MWNT/SBR (8.37). Furthermore, the fatigue life of the MMT/MWNT embedded SBR was longer than those of the MWNT composites in Figure 4(b). The growth in the fatigue cracks of composites using MMT/MWNT decreased because the MMT/MWNT fillers were uniformly dispersed in the SBR, due to the formation of a segregated network with functional groups. Another reason explaining this improvement is the excluded volume created by the micrometer scale clay clusters, which form a segregated network of nanotubes.
The thermal and electrical conductivities of the elastomer composites with different embedded materials are showed in Figure 5. The thermal conductivity of the MMT/MWNT composites at $5 \mathrm{phr}$ loading in SBR is $0.3397 \mathrm{~W} / \mathrm{mK}$, which is much higher than those of the reference composites $(0.3126)$ due to the outstanding dispersibility. Also, the electrical conductivity $(19 \mathrm{~K} \Omega$ ) of the MMT/MWNT nanocomposites is one order of magnitude higher than those of the reference composites $(612 \mathrm{~K} \Omega$ ) or the MMT composites $(912 \mathrm{~K} \Omega$ ), as shown in Figure 5(a). The intimate interfacial bonding between the MMT/MWNT and the elastomer matrix may be responsible for the superior thermal and electrical conductivities [25]. Furthermore, the fully exfoliated MMT/MWNT, and its good dispersion in the elastomer composites, enhanced the gas barrier performance of the composites. Low gas permeability can be produced by the formation of a tortuous path around the fillers in the elastomer composite, which can restrain the progression of gas molecules through the matrix. Figure 5(b) shows a comparison of the gas- permeability and relative permeability of the S-MM/elastomer composites with diverse nanomaterials. The lowest oxygen transmission rate (OTR) value, of $8.16 \times 10^{-14} \mathrm{~m}^{4} \mathrm{sec}^{-1} \mathrm{~N}^{-1}$, was obtained for the MMT/MWNT/elastomer composites. The MMT/MWNT/elastomer composites show a 33\% 




(a)

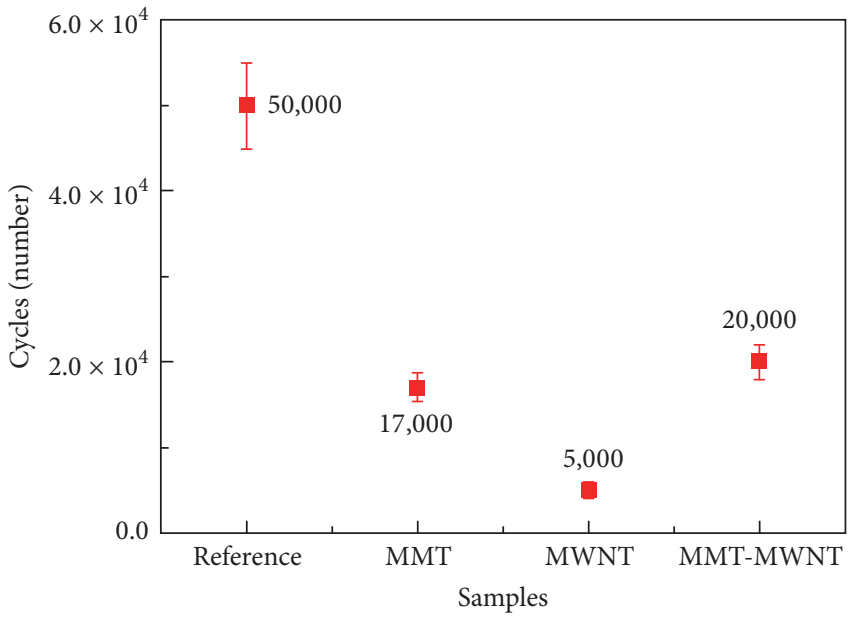

(b)

FIGURE 4: Fatigue properties of elastomer nanocomposites. (a) Fatigue properties (fatigue crack growth) of the elastomer composites with diverse fillers. (b) Fatigue properties (fatigue crack generation) of the elastomer composites with diverse fillers.

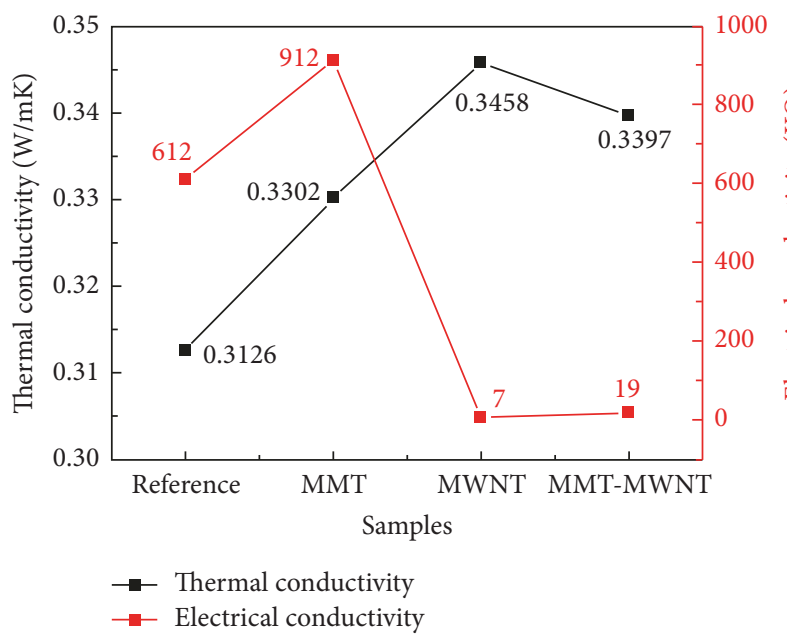

(a)



(b)

Figure 5: Thermal, electrical, and gas permeability properties of elastomer nanocomposites. (a) Thermal and electrical conductivities of the elastomer nanocomposites with diverse fillers. (b) Gas permeability and relative permeability of the elastomer composites with diverse fillers at 5 phr loading.

enhancement in barrier performance compared to the reference composites and a $27 \%$ enhancement compared to the MWNT/elastomer composites. This result may be attributed to the large sized MMT platelets and their good dispersion, produced by the mutual dispersion of the MWNT and MMT. The outstanding gas barrier behavior of the MMT/MWNT/elastomer composites is promising for applications such as tire inner liners and elastomer tubes.

The MMT/MWNT nanomaterials homogeneously dispersed in the SBR matrix produced remarkable improvements in mechanical properties such as modulus, tensile strength, and fatigue properties, even at low loadings, and these results are ascribed to the enhanced interfacial bonding with the elastomer matrix. Moreover, the incorporation of the MMT/MWNT into the SBR matrix also significantly improved thermal, electrical, and gas permeability properties. Therefore, we can confirm that our approach suggests a fascinating new potential for scalable and commercial polymer engineering.

\section{Conclusion}

In summary, we integrated hybrid nanofillers into an elastomer matrix using a simple and versatile solution mixing process. Furthermore, a novel preparation approach to produce elastomer nanocomposites with SBR improved 
mechanical, thermal, and electrical properties and gas barrier performance was developed by improving the interfacial interactions between the hybrid nanomaterials. These results showed that the hybrid nanofillers were more effective than $\mathrm{C} / \mathrm{B}, \mathrm{MWNT}$, and MMT fillers for producing elastomer composites by the extraordinary synergetic effects at low loading levels. The fabricated hybrid nanofiller and its applications as a novel networked reinforcement may open new opportunities for preparing high performance elastomer composites.

\section{Conflicts of Interest}

The author declares that they have no conflicts of interest.

\section{Acknowledgments}

The author acknowledges NEXEN Tire for fabricating rubber nanocomposites.

\section{References}

[1] Z. Tang, L. Zhang, W. Feng, B. Guo, F. Liu, and D. Jia, "Rational design of graphene surface chemistry for high-performance rubber/graphene composites," Macromolecules, vol. 47, no. 24, pp. 8663-8673, 2014.

[2] L. Bokobza, "Multiwall carbon nanotube elastomeric composites: a review," Polymer Journal, vol. 48, no. 17, pp. 4907-4920, 2007.

[3] A. Das, G. R. Kasaliwal, R. Jurk et al., "Rubber composites based on graphene nanoplatelets, expanded graphite, carbon nanotubes and their combination: A comparative study," Composites Science and Technology, vol. 72, no. 16, pp. 1961-1967, 2012.

[4] S. Araby, Q. Meng, L. Zhang, I. Zaman, P. Majewski, and J. Ma, "Elastomeric composites based on carbon nanomaterials," Nanotechnology, vol. 26, no. 11, Article ID 112001, 2015.

[5] A. M. Kucherskii, "Hysteresis losses in carbon-black-filled rubbers under small and large elongations," Polymer Testing, vol. 24, no. 6, pp. 733-738, 2005.

[6] G. Sui, W. H. Zhong, X. P. Yang, Y. H. Yu, and S. H. Zhao, "Preparation and properties of natural rubber composites reinforced with pretreated carbon nanotubes," Polymers for Advanced Technologies, vol. 19, no. 11, pp. 1543-1549, 2008.

[7] S. H. Song, H. K. Jeong, K. Y. Gu, and O.-T. Cho, "Physical and chemical characteristics of multi-walled carbon nanotube (MWCNT) with acid-treatment and coupling agent on the properties of styrene butadiene rubber (SBR)," Polymer Korea, vol. 34 , no. 2, pp. 108-115, 2010.

[8] S. Varghese and J. Karger-Kocsis, "Natural rubber-based nanocomposites by latex compounding with layered silicates," Polymer Journal, vol. 44, no. 17, pp. 4921-4927, 2003.

[9] M. Arroyo, M. A. López-Manchado, and B. Herrero, "Organomontmorillonite as substitute of carbon black in natural rubber compounds," Polymer Journal, vol. 44, no. 8, pp. 2447-2453, 2003.

[10] P. Bala, B. K. Samantaray, S. K. Srivastava, and G. B. Nando, "Organomodified montmorillonite as filler in natural and synthetic rubber," Journal of Applied Polymer Science, vol. 92, no. 6, pp. 3583-3592, 2004.
[11] E. Morales and J. R. White, "Effect of ageing on the mechanical properties and the residual stress distribution of hybrid clayglass fibre-polypropylene injection mouldings," Journal of Materials Science, vol. 44, no. 17, pp. 4734-4742, 2009.

[12] S.-H. Song, O.-S. Kwon, H.-K. Jeong, and Y.-G. Kang, "Properties of styrene-butadiene rubber nanocomposites reinforced with carbon black, carbon nanotube, graphene, graphite," Korean Journal of Materials Research, vol. 20, no. 2, pp. 104-110, 2010.

[13] D. W. McCarthy, J. E. Mark, and D. W. Schaefer, "Synthesis, structure, and properties of hybrid organic-inorganic composites based on polysiloxanes. I. Poly(dimethylsiloxane) elastomers containing silica," Journal of Polymer Science Part B: Polymer Physics, vol. 36, no. 7, pp. 1167-1189, 1998.

[14] Y. Ikeda and Y. Kameda, "Preparation of "Green" composites by the sol-gel process: In situ silica filled natural rubber," Journal of Sol-Gel Science and Technology, vol. 31, no. 1-3, pp. 137-142, 2004.

[15] L. Dewimille, B. Bresson, and L. Bokobza, "Synthesis, structure and morphology of poly(dimethylsiloxane) networks filled with in situ generated silica particles," Polymer Journal, vol. 46, no. 12, pp. 4135-4143, 2005.

[16] F. Schmitz, G. M. V. Thielen, N. Costantini et al., "Tire with a component containing carbon nanotubes," US 20110146859 A1 to Goodyear, 2011.

[17] M. Kikuchi, I. Harada, and K. Mizuno, "Orientated carbon nanotube composite, process for producing orientated carbon nanotube composite, and, produced using orientated carbon nanotube composite pneumatic tire, wheel for vehicle, tire wheel assembly and disk brake," US20060061011A1 to Bridgestone Corporation, 2006.

[18] C. A. Mitchell, J. L. Bahr, S. Arepalli, J. M. Tour, and R. Krishnamoorti, "Dispersion of functionalized carbon nanotubes in polystyrene," Macromolecules , vol. 35, no. 23, pp. 8825-8830, 2002.

[19] C. Milone, M. Dhanagopal, S. Santangelo, M. Lanza, S. Galvagno, and G. Messina, "K10 montmorillonite based catalysts for the growth of multiwalled carbon nanotubes through catalytic chemical vapor deposition," Industrial \& Engineering Chemistry Research, vol. 49, no. 7, pp. 3242-3249, 2010.

[20] C. Zhang, S. Huang, W. W. Tjiu, W. Fan, and T. Liu, "Facile preparation of water-dispersible graphene sheets stabilized by acid-treated multi-walled carbon nanotubes and their poly(vinyl alcohol) composites," Journal of Materials Chemistry, vol. 22, no. 6, pp. 2427-2434, 2012.

[21] Y.-Q. Zhao, K.-T. Lau, Z. Wang et al., "Fabrication and properties of clay-supported carbon nanotube/poly (vinyl alcohol) nanocomposites," Polymer Composites, vol. 30, no. 6, pp. 702$707,2009$.

[22] C. Tang, L. Xiang, J. Su et al., "Largely improved tensile properties of chitosan film via unique synergistic reinforcing effect of carbon nanotube and clay," The Journal of Physical Chemistry B, vol. 112, no. 13, pp. 3876-3881, 2008.

[23] S. H. Song, "Synergistic Effect of Clay Platelets and Carbon Nanotubes in Styrene-Butadiene Rubber Nanocomposites," Macromolecular Chemistry and Physics, vol. 217, no. 23, pp. 26172625,2016

[24] D. Lee, S. H. Song, J. Hwang et al., "Enhanced mechanical properties of epoxy nanocomposites by mixing noncovalently functionalized boron nitride nanoflakes," Small, vol. 9, no. 15, pp. 2602-2610, 2013. 
[25] S. Shenogin, A. Bodapati, L. Xue, R. Ozisik, and P. Keblinski, "Effect of chemical functionalization on thermal transport of carbon nanotube composites," Applied Physics Letters, vol. 85, no. 12, pp. 2229-2231, 2004. 


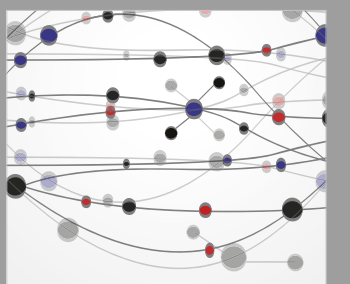

The Scientific World Journal
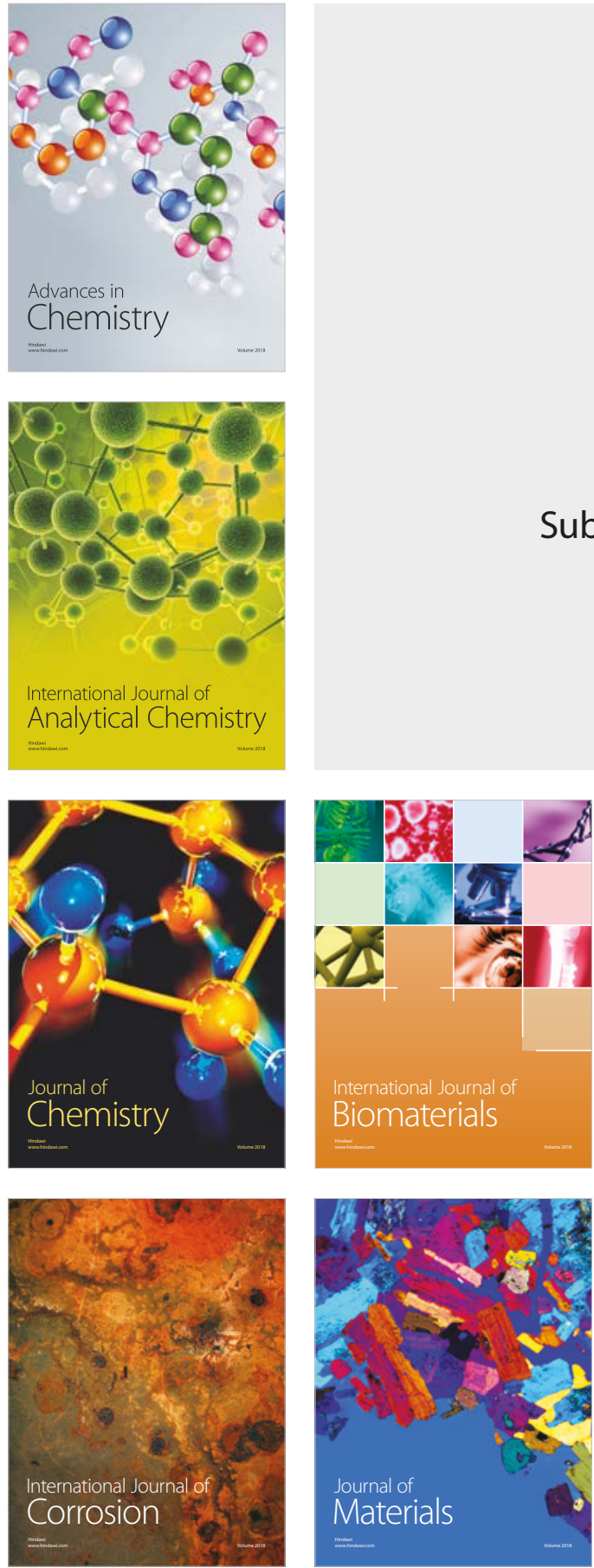

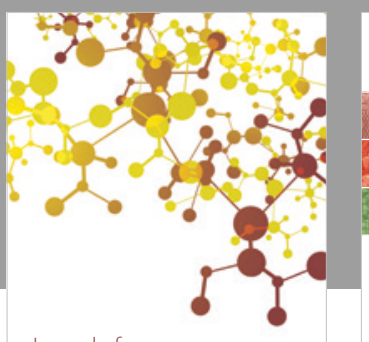

Journal of

Applied Chemistry
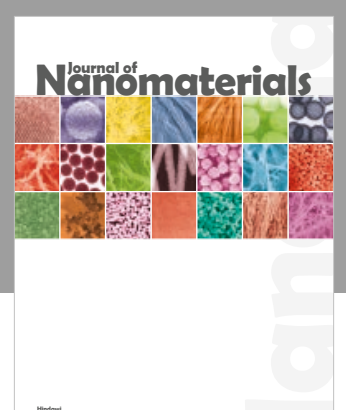

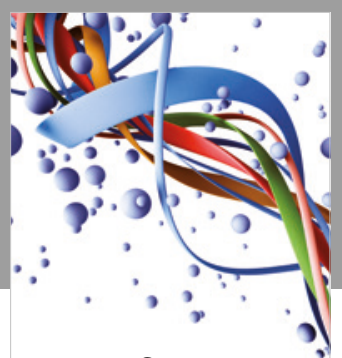

Scientifica



Polymer Science



Physical Chemistry
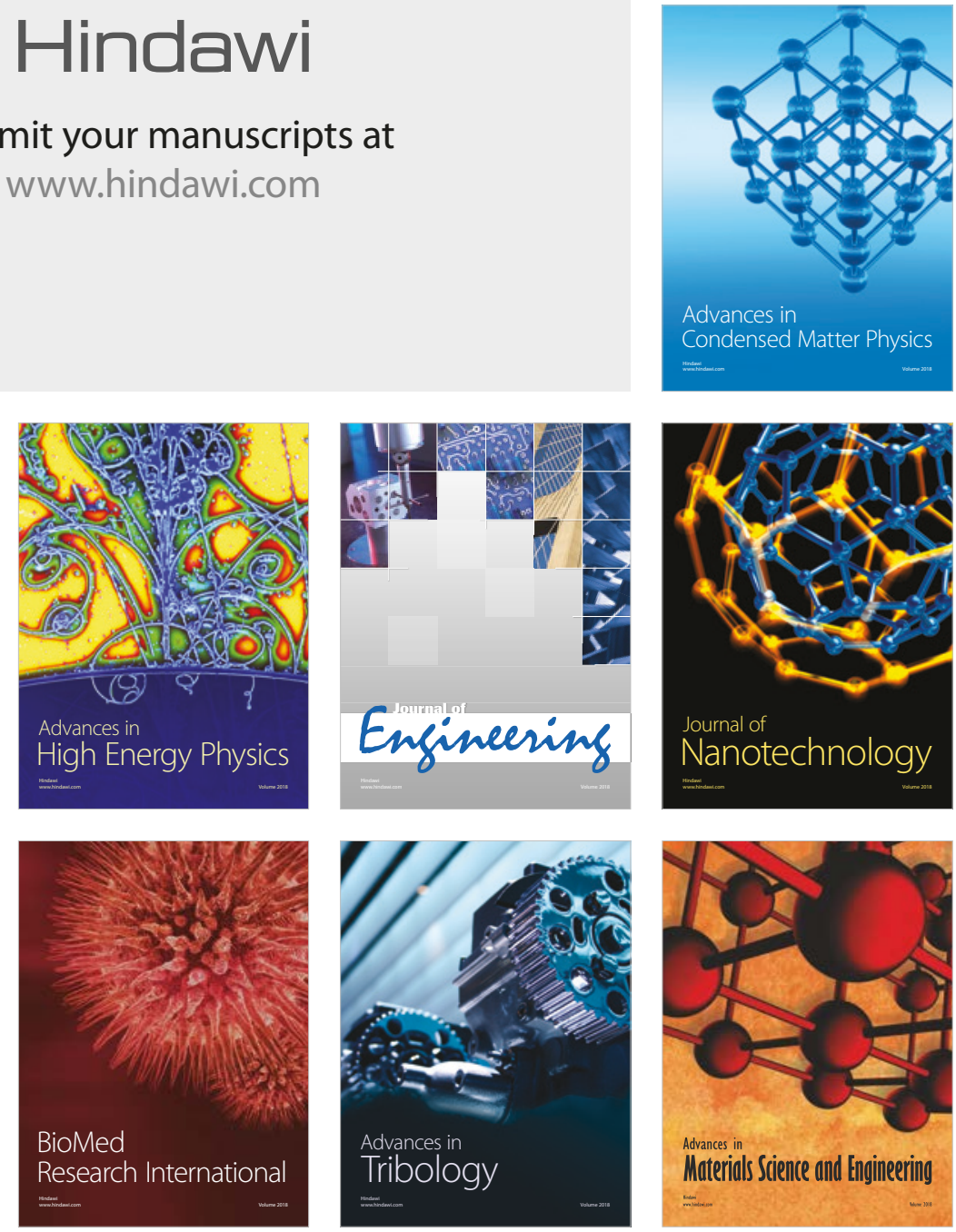